\title{
Structural Relation Between the X-Phase and Other Phases in $\mathbf{N i}_{\mathbf{2}} \mathbf{M n G a}$
}

\author{
Tomoyuki Kakeshita ${ }^{1}$, Takashi Fukuda ${ }^{1, a}$, Tomoyuki Terai ${ }^{1}$, \\ Toyotaka Osakabe $^{2}$ and Kazuhisa Kakurai ${ }^{2}$ \\ ${ }^{1}$ Department of Materials Science and Engineering, Graduate School of Engineering, \\ Osaka University, 2-1, Yamada-oka, Suita, Osaka 565-0871, Japan \\ ${ }^{2}$ Quantum Beam Science Directorate, Japan Atomic energy Agency, \\ Naka-gun, Ibaraki 319-1195, Japan \\ afukuda@mat.eng.osaka-u.ac.jp
}

Keywords: incommensurate phase, critical phenomena, neutron diffraction, ferromagnetic shape memory alloy, nickel-manganese-gallium alloy

\begin{abstract}
We have investigated stress and temperature dependences of the structure of the X-phase in $\mathrm{Ni}_{2} \mathrm{MnGa}$ to understand structural relation between the X-phase and other phases. Position and intensity of satellites of the X-phase are different from those of the intermediate (I-) phase under compressive stress, but they approach those of the I-phase with decreasing stress. That is, the structure change associated with the $\mathrm{I} \rightarrow \mathrm{X}$ transformation is discontinuous under a compressive stress, while it is continuous under zero stress. In addition, the transformation from the X-phase to the L2 $1_{1}$-type parent phase is continuous regardless of applied stress. These results strongly suggest the existence of multi-critical point in $\mathrm{Ni}_{2} \mathrm{MnGa}$. On the other hand, the transformation from the X-phase to the martensite phase is discontinuous regardless of applied stress.
\end{abstract}

\section{Introduction}

Ferromagnetic shape memory alloy $\mathrm{Ni}_{2} \mathrm{MnGa}$ exhibits a successive thermoelastic martensitic transformation. It has been widely accepted that the transformation sequence is a L21-type parent phase (P-phase) to an intermediate phase (I-phase) and then to a martensite phase (M-phase) [1 - 4]. In addition to these phases, we recently found a new phase (X-phase) by compressive tests [5], and constructed a stress-temperature phase diagram [6]. According to the phase diagram, the X-phase exists even under zero stress. That is, transformation sequence of $\mathrm{Ni}_{2} \mathrm{MnGa}$ under zero stress is $\mathrm{P} \rightarrow$ $\mathrm{X} \rightarrow \mathrm{I} \rightarrow \mathrm{M}$, being different from the sequence reported before the finding of the $\mathrm{X}$-phase. These results suggest that $\mathrm{TA}_{2}$ phonon softening of the $\mathrm{P}$-phase, which is so far considered to be one of the precursor phenomena of the $\mathrm{P} \rightarrow \mathrm{I}$ transformation $[3,4]$, is possibly related to the $\mathrm{P} \rightarrow \mathrm{X}$ transformation. Thus, the X-phase is important to understand the martensitic transformation in $\mathrm{Ni}_{2} \mathrm{MnGa}$.

Crystal structures of the M- and I-phases have been intensively investigated so far [7 -10], and recent studies suggest that they have incommensurate structures $[12,13]$. Moreover, the X-phase also has an incommensurate structure according to our previous study [14]. Thus, three different incommensurate structures (M-, I- and X-phases) come to appear in $\mathrm{Ni}_{2} \mathrm{MnGa}$. Although these incommensurate structures are possibly related to each other, structural relation among P-, X-, I- and M-phases is not clear yet.

In the present study, therefore, we investigated structural relation among these phases by measuring stress and temperature dependences of satellite reflections of the X-, I- and M-phases in $\mathrm{Ni}_{2} \mathrm{MnGa}$. 


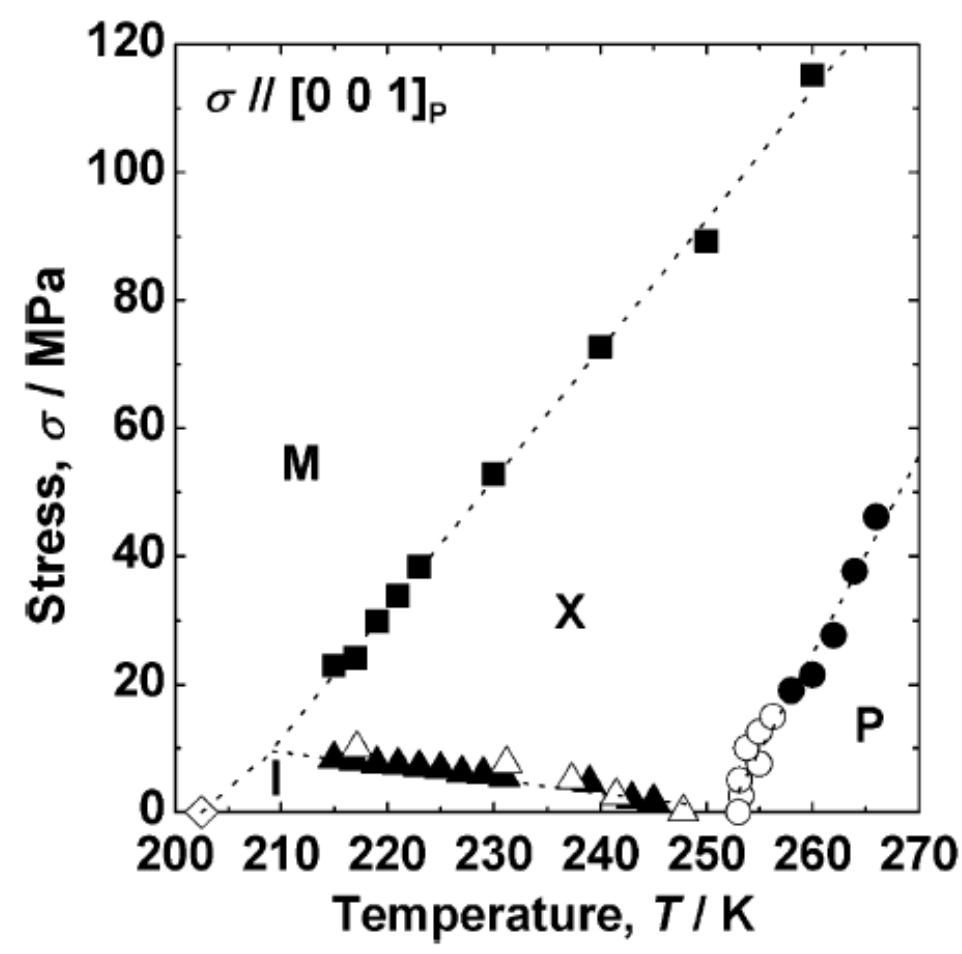

Fig. 1:

Stress-temperature phase diagram of $\mathrm{Ni}_{2} \mathrm{MnGa}$ obtained by applying compressive stress in the $[001]_{P}$ direction. (after [6])

\section{Experimental Procedure}

The specimen used in the present study is the same one used in the previous papers [13, 14]. An ingot of stoichiometric $\mathrm{Ni}_{2} \mathrm{MnGa}$ was prepared by arc-melting, and a single crystal was grown from the ingot by a floating zone method. It was heat-treated at $1173 \mathrm{~K}$ for $24 \mathrm{~h}$ for homogenization and then at $923 \mathrm{~K}$ for $24 \mathrm{~h}$ to obtain a highly ordered L $2{ }_{1}$-type structure. A specimen with a dimension of $5.0 \times$ $1.9 \times 1.9 \mathrm{~mm}^{3}$ and all edges being parallel to $<0 \quad 0 \quad 1>_{\mathrm{P}}$ was cut from the single crystal. The transformation temperature of the specimen was examined by a magnetic susceptibility measurement. The obtained transformation start temperatures are $259 \mathrm{~K}$ for the $\mathrm{P} \rightarrow \mathrm{X}$ transformation, $254 \mathrm{~K}$ for the $\mathrm{X} \rightarrow \mathrm{I}$ and $207 \mathrm{~K}$ for the $\mathrm{I} \rightarrow \mathrm{M}$. These temperatures are slightly higher than those of the specimen used to determine Fig. 1. So, the experimental conditions are determined taking the difference into consideration. Details of sample preparation are written elsewhere [13, 14].

Neutron diffraction measurements were made with a triple-axis spectrometer (TAS-1) using a non-polarized neutron beam at 2G beam-line of JRR-3 in Japan Atomic Energy Agency (JAEA). A wave length of $0.236 \mathrm{~nm}$ was selected through the neutron diffraction measurements. The horizontal collimation used was open-80'-80'-80'. A pyrolytic graphite filter was used to attenuate higher-order harmonic contaminations. Measurements were made in the temperature range of $190 \mathrm{~K} \leq \mathrm{T} \leq 260 \mathrm{~K}$ under compressive stresses of $1 \mathrm{MPa} \leq \sigma \leq 100 \mathrm{MPa}$ applied in the $\left[\begin{array}{lll}0 & 0 & 1\end{array}\right]_{\mathrm{P}}$ direction by sweeping the scattering vector $\boldsymbol{q}=\left[\begin{array}{ll}h & 2-h 0\end{array}\right]^{*} \mathrm{p}$ in the range of $-0.1 \leq h \leq 2.1$. The lowest stress of the present experiment is about $1 \mathrm{MPa}$ due to the weight of the piston used to apply external stress.

\section{Results}

Figure 2 shows stress dependence of neutron profile measured under different compressive stresses of $1 \mathrm{MPa} \leq \sigma \leq 100 \mathrm{MPa}$ at $255 \mathrm{~K}$. In the case under $1 \mathrm{MPa}$, extremely weak satellite reflection appears at an incommensurate position of $h=0.341$ (the index $h$ represents the satellite position of [ $h$ 2- $h$ $0]^{*}$ ). Although this satellite is due to the X-phase, its position is close to that of the I-phase under zero stress reported in a previous paper [13]. The reason why satellites of the X-and I-phases appear at almost the same position is discussed later. We notice in Fig. 2 that the satellite position and intensity depend significantly on stress. That is, the position drastically moves from $h=0.341$ (under $1 \mathrm{MPa}$ ) to $h=0.369$ (under $50 \mathrm{MPa}$ ), and the intensity obviously increases with increasing stress. In addition to the position and intensity, full width at half maximum (FWHM) also depends on stress. 
For example, the FWHM of the satellite reflection under $1 \mathrm{MPa}$ is about 0.016 , and that under $50 \mathrm{MPa}$ is about 0.030. Such an increase in FWHM means that the variance of modulation wave length increases by the application of external stress. Incidentally, the profile under $100 \mathrm{MPa}$ shows the coexistence of the $\mathrm{X}$ - and M-phases. This coexistence is due to the first-order nature of the stress-induced $\mathrm{X} \rightarrow \mathrm{M}$ transformation, being consistent with the stress-temperature phase diagram shown in Fig. 1.

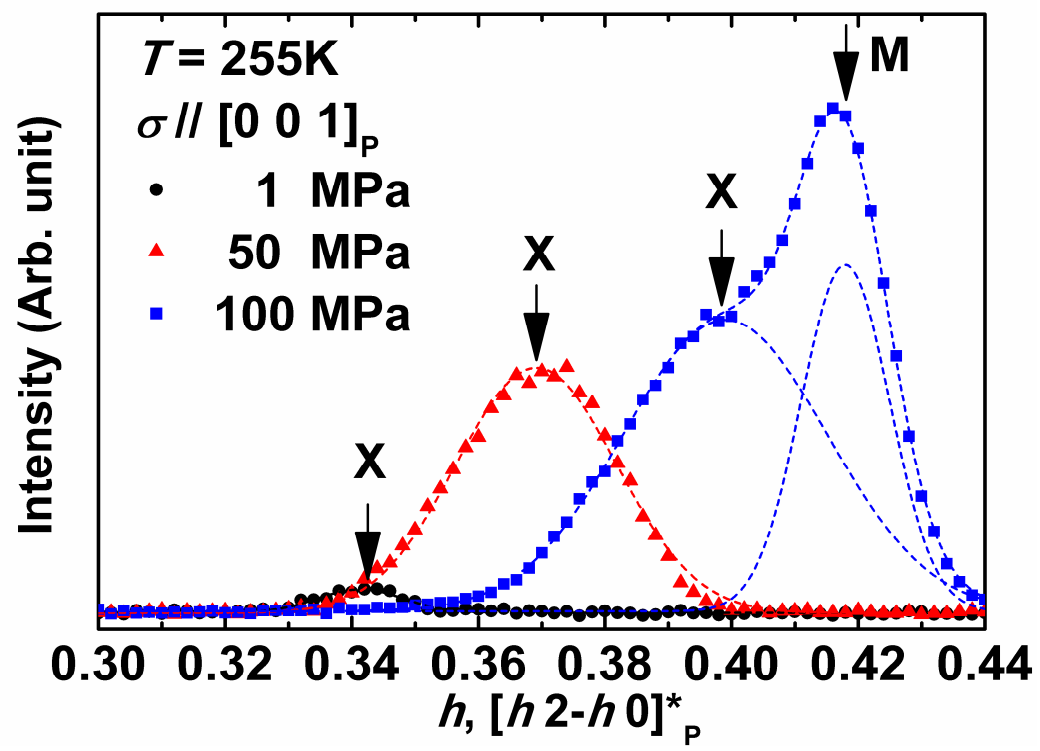

Fig. 2:

Neutron diffraction profile of the X-phase at $255 \mathrm{~K}$ under different compressive stresses. The profile under $100 \mathrm{MPa}$ comprises the X-phase and the M-phase.

Similar measurements have been made for the M-phase by fixing temperature at $190 \mathrm{~K}$. The satellite reflection of the M-phase under $1 \mathrm{MPa}$ appeared at $h=0.426$, and it shifted to $h=0.427$ under $25 \mathrm{MPa}$ and to $h=0.428$ under $40 \mathrm{MPa}$. While the satellite position depended slightly on the stress, satellite intensity and the FWHM $(\sim 0.016)$ kept almost the same values regardless of stress, meaning that the variance of modulation wave vector does not depends on stress in the M-phase.

By using the neutron profiles under various compressive stresses, we calculated lattice spacing $d$ corresponding to $\left\{\begin{array}{lll}1 & 0 & 0\end{array}\right\}_{\mathrm{P}}$ planes of the X- and M-phases. The lattice spacing of the X-phase increased from $0.581 \mathrm{~nm}$ to $0.586 \mathrm{~nm}$ with increasing stress from $1 \mathrm{MPa}$ to $85 \mathrm{MPa}$, while that of the M-phase kept almost the same value in the examined stress range. Using the stress dependence of the lattice spacing, the Young's modulus of the X-phase at $255 \mathrm{~K}$ is calculated to be about $4.8 \mathrm{GPa}$. In the calculation, we need Poisson's ratio of the X-phase, because $d$ is measured perpendicular to applied stress. We assumed that the Poisson's ratio at $255 \mathrm{~K}$ is equal to that of the P-phase at $300 \mathrm{~K}$, which is calculated to be 0.48 if we use the elastic constants reported by Worgull et al. [15]. The Young's modulus of the X-phase at $255 \mathrm{~K}$ is the same order as the value of the P-phase at $260 \mathrm{~K}, 7.0 \mathrm{GPa}$, obtained from initial slope of the stress-strain curve reported in the previous paper [6]. These low Young's moduli of the X-and P-phases possibly reflect lattice softening of the P- and X-phases near the $\mathrm{P} \leftrightarrow \mathrm{X}$ transformation temperature. This lattice softening was pointed out by Worgull et al. [15], although the X-phase was not recognized at that time.

Figure 3 shows temperature dependence of the neutron profile of the X-phase measured under a constant compressive stress of $10 \mathrm{MPa}$. In the case at $220 \mathrm{~K}$, satellite reflection of the X-phase appears at an incommensurate position of $h=0.361$. With increasing temperature, the satellite reflection obviously moves toward the nearest fundamental reflection of $\left[\begin{array}{lll}0 & 2 & 0\end{array}\right]^{*} \mathrm{p}$, and its intensity drastically decreases. In addition to the change in position and intensity, the FWHM of the X-phase decreases from 0.024 at $220 \mathrm{~K}$ to 0.020 at $260 \mathrm{~K}$.

Similar measurements have been made for the M-phase by fixing compressive stress to $1 \mathrm{MPa}$. Satellite reflection of the M-phase at $190 \mathrm{~K}$ appeared at an incommensurate position of $h=0.427$, and slightly moves to $h=0.426$ at $200 \mathrm{~K}$ and then to $h=0.424$ at $210 \mathrm{~K}$. In addition, the intensity slightly 
decreased with increasing temperature. The FWHM kept almost the same value of 0.016 regardless of temperature, which resembles the stress dependence of the FWHM described above.

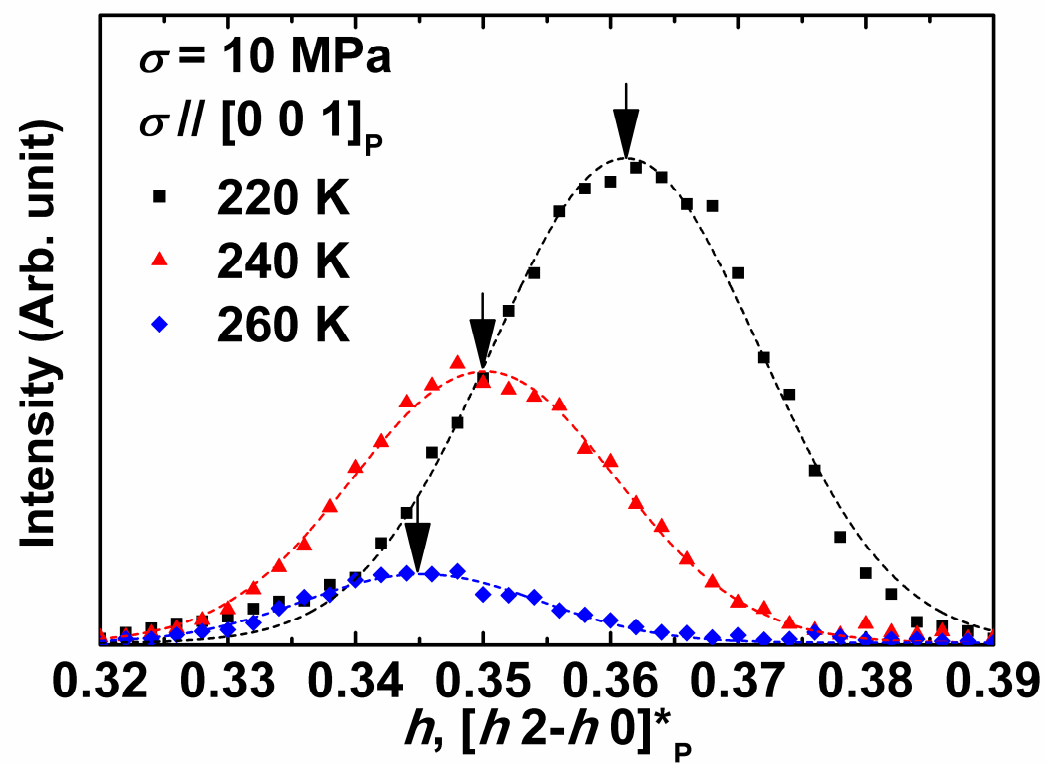

Fig. 3:

Neutron diffraction profiles of the X-phase under compressive stress of $10 \mathrm{MPa}$ at different temperature.

\section{Discussion}

In the previous section, we described the stress and temperature dependences of satellite reflections of the X-and M-phases. The obtained results of satellite positions of the X- and M-phases are summarized in Fig. 4 together with those of the I-phase under zero stress reported in the previous paper [12]. In the followings, we will discuss the structural relation between the X-phase and the other phases by referring Fig. 4.

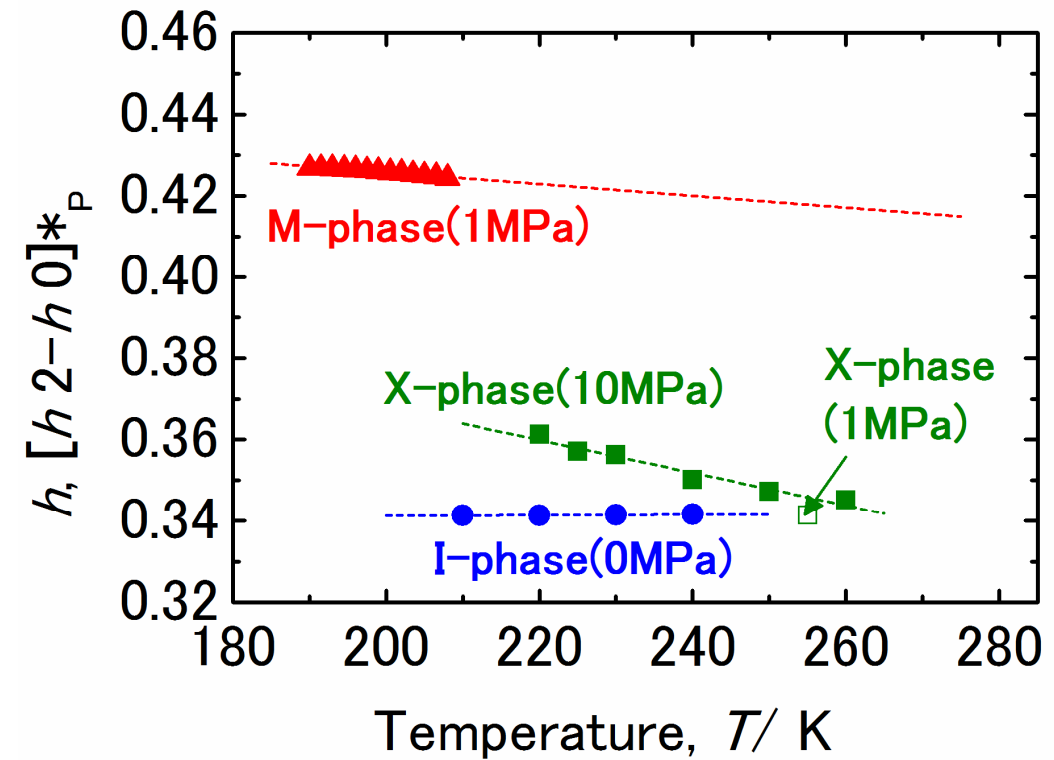

Fig. 4:

Temperature dependences of satellite position in $\mathrm{Ni}_{2} \mathrm{MnGa}$. 
First, we discuss the structural relation between the X- and M-phases. It is seen in Fig. 4 that the satellite position of the X-phase are obviously different from those of the M-phase for all the temperature region examined in the present study. In addition, the FWHM of the X-phase is nearly twice that of the M-phase as seen in Fig. 2. Consequently, the $X \rightarrow M$ transformation is a first-order one regardless of temperature. The existence of stress hysteresis in the stress-strain curves associated with the $\mathrm{X} \rightarrow \mathrm{M}$ transformation [5,6] also support the above interpretation.

Second, we discuss the structure relation between the X- and P-phases. A transmission electron microscope observation of the present alloy showed that satellite position and intensity of the $\mathrm{X}$-phase are almost the same as those of the $\mathrm{P}$-phase in the vicinity of the $\mathrm{X} \rightarrow \mathrm{P}$ transformation temperature [16]. This result suggests that the $\mathrm{X} \rightarrow \mathrm{P}$ transformation is a second-order-like one. No stress hysteresis in the stress-strain curves and no temperature hysteresis in the magnetic susceptibility curves associated with the $\mathrm{P} \leftrightarrow \mathrm{X}$ transformation $[5,6]$ also support the above interpretation.

Finally, we discuss the structure relation between the X-and I-phases. Except for in the vicinity of the $\mathrm{I} \rightarrow \mathrm{X}$ transformation temperature under zero stress, $T_{0}^{\mathrm{I} \rightarrow \mathrm{X}}$, the $\mathrm{I} \rightarrow \mathrm{X}$ transformation is a first-order one. This is because the I- and X-phases coexist in association with the transformation and a hysteresis exists for the transformation $[6,14]$. In the vicinity of $T_{0}{ }^{\mathrm{I} \rightarrow \mathrm{X}}$, however, the transformation is continuous as seen in Fig. 4. That is, the satellite position of the X-phase approaches that of the I-phase on approaching $T_{0}{ }^{\mathrm{I} \rightarrow \mathrm{X}}$. In addition, the intensity of the I-phase at zero stress approaches that of the X-phase at $1 \mathrm{MPa}$ on approaching $T_{0}^{\mathrm{I} \rightarrow \mathrm{X}}$. Therefore, the satellite reflection of the X-phase under zero stress is indistinguishable from that of the I-phase. This is the reason why the X-phase, which exists even under zero stress, has not been found by means of neutron, X-ray and electron diffraction experiments reported so far.

From the above results, it is most likely that $\mathrm{Ni}_{2} \mathrm{MnGa}$ has a multi-critical point like the one observed in $\mathrm{SrTiO}_{3}$ [17]. That is, second-order-like $\mathrm{P} \rightarrow \mathrm{X}$ transformation is followed by second-order-like $\mathrm{X} \rightarrow$ I transformation and these two transformations occur almost at the same temperature.

\section{Conclusions}

Stress and temperature dependences of the structure of the $\mathrm{X}$-phase in $\mathrm{Ni}_{2} \mathrm{MnGa}$ have been investigated by neutron diffraction measurements under compressive stress in order to clarify structural relation among the P-, I-, M- and X-phases. The structure of the X-phase in $\mathrm{Ni}_{2} \mathrm{MnGa}$ depends strongly on stress and temperature compared with that of the M-phase and/or the I-phase. In association with the $\mathrm{X} \rightarrow \mathrm{M}$ transformation, both the position and intensity of the satellite reflection changes drastically regardless of stress and temperature. In association with the $\mathrm{X} \rightarrow \mathrm{P}$ transformation, the intensity of the satellite reflection fades gradually. In association with the I $\rightarrow \mathrm{X}$ transformation, the position and intensity of the satellite changes drastically when the transformation occur under compressive stress, while they changes continuously when it occurs under zero stress.

\section{Acknowledgements}

This study was supported by The Global COE Program (Project: Center of Excellence for Advanced Structural and Functional Materials Design) from MEXT, Japan. This work was performed under the Common-Use Facility Program of JAEA.

\section{References}

[1] P. J. Webster, K. R. A. Ziebeck, S. L. Town and M. S. Peak: Phil. Mag. B Vol. 49 (1984), p.295 
[2] V. V. Kokorin, V. A. Chernenko, E. Cesari, J. Pons and C. Segui, J. Phys.: Condens. Matter: Vol. 86457 (1996), p. 6457

[3] A. Zheludev, S. M. Shapiro, P. Wochner, A. Schwartz, M. Wall and L. E. Tanner: Phys. Rev. B Vol. 51 (1995), p. 11310

[4] A. Planes, E. Obradó, A. G. Comas and L. Manõsa: Phys. Rev. Lett Vol. 79 (1997), p. 3926

[5] J. H. Kim, T. Fukuda and T. Kakeshita: Scripta Mater. Vol. 54 (2006), p. 585

[6] H. Kushida, K. Hata, T. Fukuda, T. Terai and T. Kakeshita: Scripta Mater. Vol. 60 (2009), p. 96

[7] V. V. Martynov and V. V. Kokorin: J. Phys. (France) III 2, (1992), p.739

[8] A. Zheludev, S. M. Shapiro, P. Wochner and L. E. Tanner: Phys. Rev. B Vol. 54 (1996), p. 15045.

[9] J. Pons, V. A. Chernenko, R. Santamarta and E. Cesari: Acta Mater. Vol. 48 (2000), p. 3027

[10] P. J. Brown, J. Creangle, T. Kanomata, M. Matsumoto, K. U. Neumann, B. Ouladdiaf and K. R. A. Ziebeck: J. Phys.: Condens. Matter Vol. 14 (2002), p. 10159

[11] T. Ohba, N. Miyamoto, K. Fukuda, T. Fukuda, T. Kakeshita and K. Kato: Smart Mater. Struct. Vol. 14 (2005) p. S197

[12] L. Righi, F. Albertini, L. Pareti, A. Paoluzi and G. Calestani: Acta Mater. Vol. 55 (2007), p. 5237

[13] H. Kushida, K. Fukuda, T. Terai, T. Fukuda, T. Kakeshita, T. Ohba, T. Osakabe, K. Kakurai and K. Kato, Eur. Phys. J. Special Topics, Vol. 158 (2008), p. 87

[14] H. Kushida, T. Terai, T. Fukuda, T. Kakeshita, T. Osakabe and K. Kakurai: Scripta Mater. Vol. 60 (2009), p. 248

[15] J. Worgull, E. Petti and J. Trivisonno: Phys. Rev. B Vol. 54 (1996), p.15695

[16] T. Fukuda, H. Kushida, M. Todai, T. Kakeshita and H. Mori: Scripta Mater. DOI: 10.10.16/j.scriptamat.2009.04.046

[17] K. A. Müller, W. Berlinger and J. C. Slonczewski, Phys. Rev. Lett., Vol. 25 (1970), p. 734 